\title{
Effect of Fatigue on Knee Kinematics During Countermovement Jumps in Female Students of Physical Education With Valgus Knee Deformity
}

Najmeh Sharifi ${ }^{1}$, Mohammadreza Amir-Seyfaddini' ${ }^{1}$, Mohammadtaghi Amiri-Khorasani ${ }^{1 *}$

1. Department of Sports Biomechanics, Faculty of Physical Education, Shahid Bahonar University of Kerman, Kerman, Iran.

Citation: Sharifi N, Amir-Seyfaddini MR, Amiri-Khorasani MT. Effect of Fatigue on Knee Kinematics During Countermovement Jumps in Female Students of Physical Education With Valgus Knee Deformity. Iranian Rehabilitation Journal. 2016; 14(2):107-114. https://doi.org/10.18869/nrip.irj.14.2.107

https://doi.org/10.18869/nrip.irj.14.2.107

Article info:

Received: 14 Dec. 2015

Accepted: 31 Mar. 2016

\section{Keywords:}

Valgus knee deformity, Counter movement jump, Kinematics, Fatigue

\begin{abstract}
Objectives: Skeletal-muscular disorders affect the biomechanics of the human body, especially the lower extremity, with a negative effect on the biomechanics of exercise. The main aim of this study was to evaluate the effect of fatigue on the kinematic variables of knee joints during countermovement jumps in female students of physical education with valgus knee deformity.

Methods: Fifteen subjects with valgus knee deformity and 15 healthy subjects without any damage in the lower extremities, particularly in the knees, volunteered to participate in this research. The healthy subjects were identical to those with valgus knee deformity in terms of demographic factors. All of them performed 3 countermovement jumps before and after the fatigue program. To create fatigue, a functional fatigue program was utilized, and the Borg scale was used to determine the level of fatigue in the patients.
\end{abstract}

Results: The findings showed that maximum angular velocity and angular displacement significantly decreased in both the groups, after the induction of fatigue. The difference in the valgus degree, before and after fatigue, was greater in patients with valgus deformity as compared to the healthy subjects.

Discussion: According to the results, the effects of fatigue on the kinematic variables in patients with valgus knee deformity are greater than on the healthy people. This could have been caused by the changes in the lower extremities and muscle length in patients with valgus knee deformity.

\section{Introduction}

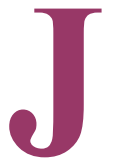

ump is considered as a basic movement skill and a predictive factor of success in explosive exercise movements [1]. A countermovement jump is often initiated by the downward movement of the body. A coun- termovement follows two major objectives: first, it guides the body in a better position to start the pushing forward step and second, it creates a stretch-shortening effect [2]. A person can jump 3-6 cm higher by a countermovement jump through a stretch-shortening cycle, where the muscles are positioned in the desired direction before the contraction and involved in the primary traction in the

* Corresponding Author:

Mohammadtaghi Amiri-Khorasani, PhD

Address: Department of Sports Biomechanics, Faculty of Physical Education, Shahid Bahonar University of Kerman, Kerman, Iran.

Tel: +98 (913) 1999143

E-mail:amirikhorasani@uk.ac.ir 
opposite direction [2]. The stretch-shortening cycle in a countermovement jump consists of three phases: 1) The eccentric phase: the pelvic flexion caused by gluteus and knee flexion hamstrings driven by quadriceps are done controllably 2) Attenuation or depreciation phase: the second moving moment when an eccentric reduction leads to a concentric enhancement; 3) Concentric phase: hip and knee extensions are performed explosively [3, 4]. Note that the mechanism of a countermovement jump is caused to some key parameters which include [1] the muscle activity which will be caused to work in lower extremity [2], the ground reaction force [3], and the displacement of the center of gravity [2].

One of the factors likely to affect the control of lower extremity joints is muscle fatigue. A combined fatigue condition occurs based on the physiological mechanisms at the central and peripheral levels that affect neuromuscular afferent pathways, causing a proprioceptive defect. This is evident as delayed muscle response. McArden (1998) stated that fatigue results from the discontinuation of the chain of events from the central nervous system to muscle fibers. Fatigue is defined in different ways; however, its best definition has been presented by Vince and Enoka (1992). Vince defined fatigue as an event, in which muscle tissue cannot support the metabolism of contractile elements caused by anemia or local discharge of each metabolic material. Enoka considered fatigue as a serious effect imposing a deficit on the elements, which includes both an increase in the sense of trying to apply a demanded power and a final disability to reproduce force. It seems that a combination of these two definitions accounts for the most appropriate approach to the definition of fatigue [5].

Musculoskeletal disorders affect the biomechanics of the human body, especially the lower extremity with a negative effect on the biomechanics of exercise. If a person has a deformity in the knee joint, fatigue seems to have a greater impact on him or her. In the case of knee abnormality, there is a change in the load amount resulting from the weight on the inside and outside of the knee. Also, due to a change in the gravity line position and exertion of an abnormal pressure on different parts of the joint, the knee will be prone to premature wear, and thus, premature arthritis can be expected.

Among the most common deformities of the knee joint, genu valgus and genu varus deformities, also known as valgus and genu leg, can be mentioned. These are more common among women than in men [6]. Such changes in the lower extremities can lead to a disruption of the gravity line proportional to the level of support and eventually cause a major shift in personal performance [7]. This deformity is a disorder, in which the genu valgus deformity displaces the foot gravity line towards the outer part of the knee and enhances the pressure in this area [19].

Benjamin et al. (2005) examined the effect of fatigue on the kinematic variables of hip and knee joints in a single leg landing and found a decrease in the knee angular displacement after flexion fatigue in both the genders [8]. Santamaria et al. (2010) investigated the effect of fatigue on the biomechanics of the lower extremity during a single leg landing and expressed a significant reduction of maximum knee valgus and flexion after fatigue in both male and female athletes. Also, internal rotation of the leg, angular displacement, and knee flexion time for maximum angular displacement were found to be less in women than men after the induction of fatigue [9].

Chappell et al. (2007) examined the effects on the kinetic and kinematic characteristics of the knee joint and observed a significant decrease in the knee flexion angle, increased valgus torque, enhanced proximal tibia anterior shear force, and reduced angular velocity during leg landing [10]. Since knee joint abnormalities can cause problems, the cost of treatment in daily life needs to be increased. According to the issues raised, our research question states whether fatigue has any effect on the kinematic variables (angular displacement of the knee at the eccentric phase, angular velocity at the concentric stage and changes in knee valgus) of patients with a valgus deformity condition. This leads to the consideration of fatigue as a risk factor for the exacerbation of the mentioned disorder.

Therefore, the aim of this study was to evaluate the effect of fatigue on the kinematic variables during a countermovement jump in healthy female students of physical education and those with knee valgus deformity.

\section{Methods}

\section{Subjects}

Thirty physical education students of the Shahid Bahonar University of Kerman were purposefully divided into two equal groups of healthy people and those with knee valgus deformity, as the statistical community. Similar subjects were selected based on age, height and body mass to avoid any study bias.

\section{Procedure}

At first, each subject was given a questionnaire of individual characteristics and asked to respond to the ques- 
tions. People who had no histories of fracture or surgery in the lower extremities were eligible for the test and participated in the research. First, the subjects with valgus deformity were selected, and after testing this group, the healthy group was chosen.

To determine the extent of abnormalities in the knee, the distance between the two lateral malleoli of ankles was measured by caliper while standing and without any pressure on the internal epicondyles of the thighs when touched together. After the desired subject had been placed in the group of knee valgus deformity, its structural or functional state was examined. Those with structural valgus deformity were not included in the research. All the examinations were done under the supervision of an MA expert in sports pathology [20].

To perform a three-dimensional (3D) record of the subjects' countermovement jumps before and after the fatigue protocol, a 3D Optoelectronic Motion Analysis system was employed by using six cameras. This system is capable of performing a $3 \mathrm{D}$ video photography up to 900 frames per second. For this study, the frequency of the cameras was considered to be $120 \mathrm{~Hz}$ [11, 12]. Calibration of the cameras was conducted in such a way that the X-axis was located perpendicular to his or her frontal plane (otherwise the knee angles could not be obtained exactly in the frontal plane).

The calibrated volume was in a way that it could completely cover the subject during the countermovement jump. This volume included a length of $2.5 \mathrm{~m}$, width of $1.5 \mathrm{~m}$, and height of $2.5 \mathrm{~m}$. Sorting of the cameras was so that every marker was visible at least by two cameras in every moment. Three patio reflective markers were placed in the desired positions. These sites included the anterior and medial shank in the distal part of the tibial bone, tibial tuberosity and the midpoint, i.e. the line that connects the anterior superior iliac spine to the patella of the dominant leg $[11,12]$.

On the day of the study, to avoid any injury to the subjects while performing the countermovement jumps and fatigue protocol, the participants did a 10-minute warmup including jogging and stretching of the lower body. After being prepared and placed in the desired position, each subject performed three countermovement jumps before and after the fatigue protocol while his or her hands were on the waist. A 5-second rest was allowed before each attempt. The mean counter-movement jump data before and after the fatigue protocol were recorded. The fatigue protocol includes a series of jumping and running on a round trip. Each participant did 15 maxi- mum drop jumps from a height of $40 \mathrm{~cm}$ and 15 maximum counter-movement jumps with a 10 -second rest in between. After 20 seconds of rest, he or she ran an $8 \mathrm{~m}$ track on a round trip for 15 seconds [13].

To ensure the subjects' fatigue, Borg's RPE-scale was used. At the beginning and end of the fatigue protocol, each subject was asked to express his or her true feelings about the intensity of the activity. The range of scores on this scale was between a minimum of 6 (very comfortable) and a maximum of 20 (very heavy). The data recorded were analyzed by using CORTEX software, version 2.5. In order to eliminate the noise caused by the movements of the markers, Butterworth low-pass filters with a frequency of $6 \mathrm{~Hz}$ were utilized.

\section{Statistical analysis}

Data was analyzed by using SPSS software, version 21 . First, the normality of the statistical community was evaluated through Shapiro test. In this research, to describe the data and assess the study variables between the two groups, i.e. comparing the experimental and control groups before and after the fatigue protocol, and descriptive statistics, paired t-test, and independent t-test at a significance level of $\mathrm{P}=0.001$ were used.

\section{Results}

To assimilate the two groups, an independent t-test was utilized after establishing the normal condition. The results demonstrated no statistically significant difference between the measured parameters (except for the distance between the lateral malleoli), and thus, the healthy subjects were assimilated with those with knee valgus deformity based on demographic factors (age, height and body mass). No significant difference was observed in the distance between the lateral malleoli regarding both the healthy patient groups $(\mathrm{P}<0.05)$.

The statistical results were indicative of a significant difference between before and after fatigue application in healthy volunteers, based on the maximum angular velocity of the knee extension $(\mathrm{P}<0.05)$. The same significant difference was found in the people with knee valgus deformity before and after fatigue for the mentioned test $(\mathrm{P}<0.05)$. However, no significant difference was discovered between the healthy subjects and those with knee valgus deformity based on the maximum angular velocity after the fatigue protocol $(\mathrm{P}>0.05)$.

The difference between before and after the fatigue protocol in the healthy subjects was significantly based 
on the maximum angular displacement $(\mathrm{P}<0.05)$. Again, the same significant difference was found in those with knee valgus deformity before and after fatigue for the mentioned test $(\mathrm{P}<0.05)$. No significant difference was discovered between the healthy subjects and those with knee valgus deformity based on the maximum angular displacement after the fatigue protocol $(\mathrm{P}>0.05)$.

The statistical results indicated that there was a significant difference in the knee valgus angle in the healthy volunteers before and after fatigue induction $(\mathrm{P}=0.001)$, and a similar condition was found for those with knee valgus deformity $(\mathrm{P}=0.001)$. In the examination of the changes in the knee joint valgus before and after fatigue, a significant difference was seen between the healthy people and those with knee valgus deformity $(\mathrm{P}=0.001)$.

\section{Discussion}

Statistical tests represented significantly reduced kinematic variables (maximum angular velocity, angular displacement, and valgus changes) in both the groups after the fatigue program. No significant difference was observed when comparing the kinematic variables (maximum angular velocity and angular displacement) between the healthy group and that of knee valgus deformity after the fatigue program. Moreover, the statistical tests displayed a significantly increased degree of valgus angle in both the groups after the fatigue program. They further exhibited a greater degree of knee valgus angle in people with knee valgus deformity before and after fatigue compared to the healthy subjects. According to the results, the effects of fatigue on kinematic variables in patients with knee valgus deformity are more than those of the healthy people, and this can be caused by the changes in the lower extremities in the mentioned patients and a subsequent change in their muscle lengths.

The results of this research were consistent with those of Benjamin et al. (2004) and Santamaria et al. (2010) regarding the kinematic variables of maximum angular velocity and angular displacement. However, these findings were in contrast with the findings of Deborah King et al (2006). Santamaria et al. (2010) evaluated the effect of fatigue on the biomechanics of the lower extremity during single-leg landing and expressed a significantly reduced maximum knee valgus, angular displacement, and knee flexion in male and female athletes after fatigue. Furthermore, internal leg rotation, angular displacement, and knee flexion time for the maximum angular displacement were found to be less in women than men after fatigue, which was congruent with the results of the current research [9].
Benjamin et al. (2005) evaluated the impact of fatigue on the hip joint and knee kinematic characters of 30 healthy subjects (15 males and 15 females) during single-leg landing. They showed a decreased flexion and angular displacement variables after fatigue program that were consistent in both the genders [8].

Deborah King (2006) studied the impact of fatigue on the kinematics of lower extremity joints in 20 athletes (10 men and 10 women) during jumping-landing and found decreased ankle inversion, increased knee flexion, reduced dorsiflexion, and an enhanced knee angular velocity after fatigue program in the case of female athletes compared to the male counterpart. The results of these two studies are inconsistent that can be reviewed based on the fatigue programs and testing methods [23].

The findings of this research were in agreement with those of Kernozek et al. (2009), Michael et al. (2003) Melniczek et al. (2007), and Chappell et al. (2004) but not with those of Benjamin et al. (2004) and Santamaria et al. (2010) based on changes in valgus angle (2009). The results of the study by Michael et al. (2003) on the effects of fatigue on the frontal landing movements showed that fatigue resulted in increased valgus angle. The mentioned result is in line with that of the current study. The only difference is that a local and isometric fatigue program was used with less generalizability in this research compared to the functional fatigue programs with sporting conditions [21].

The results obtained by Kernozek et al. (2008) showed that fatigue with squat repetitive movements to exhaustion could increase knee valgus. Although the mentioned study has employed repetitive movements to create fatigue and exhaustion without a functional fatigue program, results similar to this research have been obtained [14]. Chappell et al. (2007) examined the kinetic and kinematic impacts of fatigue on the knee on 20 athletes (10 men and 10 women) during a stop and jump. The fatigue in both groups increased valgus angle that is in line with the results of this study [10].

Benjamin et al. (2005) evaluated the impact of fatigue on the hip joint and knee kinematic characters in 30 healthy subjects (15 males and 15 females) during single-leg landing. Both genders exhibited neutral conditions (reduced valgus angle) after fatigue in a way that the peak knee valgus angle was reduced in both the groups. The mentioned study suggested the reduction in the valgus degree after fatigue was an attempt to keep the knee within the frontal plane, which is in contradiction with the results of the present study probably due to the 
fatigue programs and testing methods [8]. Santamaria et al. (2010) tested the effect of fatigue on the biomechanics of the lower extremity during single-leg landing and their results showed a significant reduction in maximum knee valgus in male and female athletes after fatigue, which contradicts with the findings of this study [9].

All voluntary activities result from a complex process, in which the brain, spinal cord, peripheral nerves, muscles and joints are involved. Damages to any of these organs can lead to malfunctions, especially in sports performance. A factor that is likely to affect control of the lower limb joints is neuromuscular fatigue. Fatigue occurs in a combination of physiological mechanisms on the central and peripheral levels affecting neuromuscular afferent pathways and leading to an impaired proprioception. This effect on the neuromuscular efferent pathways is evident as a delayed muscle response [15].

When the system is under pressure, the preliminary contraction of muscles happens to tighten the joints as a supportive response to prevent the damage. This contraction also causes joint stability to prevent collapse (irreversible condition). The overall effect of this mechanism is effective in the neuromuscular control and joint stability [16]. In addition, the dynamic body stability or any special joint such as the knee is conditioned by the neuromuscular control of all parts of the body involved in the movements. Reduced neurologic function over a long-term activity logically endangers lower limb joints, especially the knee. The mentioned studies concluded that the fatigue caused by increasing knee movements in the frontal and transverse planes could be a mechanism for a knee injury, especially the anterior cruciate ligament. Fatigue drains the energy sources of the body [17].

It has been observed that the degrees and severities of damages are higher at the end of a game, which reflects the effect of fatigue on the neuromuscular system [18]. In the knee valgus deformity, the muscles and joints of the lower limbs have lost their natural states, and the results may affect the desired performance. The congenital and abnormal biomechanical malformations could cause one-sided pressure on the tissues. These abnormalities cause secondary deformations of the leg, resulting in the disruption of the natural biomechanics of the knee joint, which further becomes prone to damages.

In the description of the results of this study, it should be noted that the joints were first bent during the eccentric phase of the countermovement jump, and thus, the range of motion to be driven forward increases.
In fact, the eccentric phase of the countermovement jump accounts for the basic functions of muscles and eccentric extensor contractions of the hip, knee and ankle. These extensor contractions are called pre-tensions because tensions in the muscles occur before using these muscles in a jump during the phase of being driven forward. Pre-tension in the muscle before it shortens causes an increase in the force that the muscle is able to produce. The faster the tension, lower is the pre-tension latency and less shortening occurs, which results in the production of more muscle force.

It is important that the countermovement jump occurs quickly and minimum delay happens between the end of countermovement jump and beginning of the phase of being driven forward [2]. If the muscles are fatigued, they are unable to carry out their tasks while the fatigue of muscles around the joint inhibits the neuromuscular joint feedback system and causes the mechanical receptors to work improperly. Fatigue reduces the activities of joint receptors, muscle spindles, and Golgi tendon organs, leading to defects in the muscle receptors. Moreover, fatigue results in the depletion of body's energy resources [16].

It can be concluded that fatigue causes a decrease in the tension forces of muscles and the energy stored in them in the pre-tensioning eccentric phase, leading to less muscular force production for the stage of being driven forward. Additionally, the slow countermovement jumps and much delay between the end of countermovement and beginning of countermovement are resulted, leading to a reduction in the joint angular velocity tending to open in the phase of being driven forward in countermovement jumps. It further results in not reaching a maximum height [2].

Such congenital and abnormal biomechanical postural abnormalities could cause one-sided pressure on the tissues. Due to the change in the direction of lower extremities, the compressive and tensile forces on the outer and inner parts of the joint are exerted in people with knee valgus deformity, respectively [19]. The mentioned people have less angular displacements and velocities compared to the healthy people, leading to a reduced efficiency in the eccentric and concentric phases of countermovement jump. Meanwhile, the effect of fatigue on people with knee valgus deformity is more complicated than in the healthier ones.

An altered neuromuscular control caused by fatigue might appear through the changes in electromyography, joint kinematics, and ground reaction force. The reduced kinematic variables observed in the knee are representative of a change in the control of lower limb muscles. 
These changes reflect the changes in the contraction patterns of the muscles around the knee. Decreased neuromuscular control of the knee joint reduces joint stability and increases the load imposed on the knee and subsequently the risk of ligament injury. The past researches have shown that when hip quadriceps contract eccentrically and are combined with knee valgus force, the possibility of the anterior cruciate ligament extension increases. The athletes' appropriate neuromuscular controls reduce the incidence of anterior cruciate ligament injury [22].

\section{Conclusion}

The results of the present study revealed that functional fatigue could reduce maximum angular displacement and angular velocity of the knee joint and enhance valgus angle in both the healthy subjects and those with knee valgus deformity while performing the countermovement jumps. Accordingly, it can be said that the identification of risk factors in different groups can prevent any damages and their consequences. Fatigue is considered to be a risk factor for sports activities, especially in the healthy individuals. According to the results of this research, it can be said that fatigue reduces performance efficiency and sports skills, which possesses risk for the individuals with knee valgus deformity.

The amount of kinematic variables of maximum angular velocity and angular displacement of the knee joint have been less in patients with knee valgus deformity compared to the healthy individuals before and after fatigue. Furthermore, the differences in the valgus angle of the knee joint have been greater in patients with knee valgus deformity compared to the healthy people before and after fatigue, which can be due to the changes in the lower limb direction and muscle length in the mentioned patients.

\section{Acknowledgments}

We thank the subjects who volunteered to participate in this study. In addition, this paper is the result of Najme $h$ Sharifi's Master of Science desertation in Sports Biomechanics at department of Sports Biomechanics, Shahid bahonar University of Kerman.

\section{Conflict of Interest}

The authors declared no conflict of interests.

\section{References}

[1] Colvin WW. The mechanics of the vertical jump: Exercise physiology laboratory [PhD dissertation]. California: California State University; 1999.

[2] Grimshaw P. Sport and exercise biomechanics. New York: Taylor \& Francis Group; 2006

[3] Taube W, Leukel C, Gollhofer A. How neurons make us jump: the neural control of stretch-shortening cycle movements. Exercise \& Sport Sciences Reviews. 2012; 40(2):106-15. doi: 10.1097/jes.0b013e31824138da

[4] James RC, Dufek JS, Bates BT. Effects of stretch shortening cycle exercise fatigue on stress fracture injury risk during landing. Research Quarterly for Exercise \& Sport. 2006; 77(1):1-13. doi: 10.1080/02701367.2006.10599326

[5] Enoka RM, Stuart DG. Neurobiology of muscle fatigue. Journal of Applied Physiology. 1992; 72(5):1631-648. doi: 10.1007/978-1-4899-1016-5_39

[6] Kendall FP, McCreary EK, Province PG, Rodgers MM, Romanin WA. Muscles testing and function with posture and pain. $5^{\text {th }}$ ed. Baltimore: Williams \& Wilkins; 2005.

[7] Felson DT, Goggins J, Niu J, Zhang Y, Hunter DJ. The effect of body weight on progression of knee osteoarthritis is dependent on alignment. Arthritis \& Rheumatism. 2004 50(12):3904-909. doi: 10.1002/art.20726

[8] Benjaminse A, Habu A, Sell TC, Abt JP, Fu FH, Myers JB, et al. Fatigue alters lower extremity kinematics during a singleleg stop-jump task. Knee Surgery, Sports Traumatology, Arthroscopy. 2008; 16(4):400-07. doi: 10.1007/s00167-007-0432-7

[9] Santamaria LJ, Webster KE. The effect of fatigue on lowerlimb biomechanics during single-limb landings: a systematic review. Journal of Orthopaedic \& Sports Physical Therapy. 2010; 40(8):464-73. doi: 10.2519/jospt.2010.3295

[10] Chappell JD, Creighton RA, Giuliani C, Yu B, Garrett WE. Kinematics and electromyography of landing preparation in vertical stop-jump risks for noncontact anterior cruciate ligament injury. American Journal of Sports Medicine. 2007; 35(2):235-41. doi: 10.1177/0363546506294077

[11] Moreno CC, Mendes LA, Lindquist AR. Effects of treadmill inclination on the gait of individuals with chronic hemiparesis. Archives of Physical Medicine \& Rehabilitation. 2011; 92(10):1675-80. doi: 10.1016/j.apmr.2011.05.016

[12] Munro A, Herrington L, Comfort P. Comparison of landing knee valgus angle between female basketball and football athletes: Possible implications for anterior cruciate ligament and patellofemoral joint injury rates. Physical Therapy in Sport. 2012; 13(4):259-64. doi: 10.1016/j.ptsp.2012.01.005

[13] Bide PS. Fatigue related gender differences in the biomechanics of drop jumps [PhD dissertation]. Miami: Miami University.

[14] Kernozek TW, Torry MR, Iwasaki M. Gender differences in lower extremity landing mechanics caused by neuromuscular fatigue. American Journal of Sports Medicine. 2008; 36(3):55465. doi: $10.1177 / 0363546507308934$

[15] Tamari K, Tinley P, Briffa K, Aoyagi K. Ethnic-, genderand age-related differences in femorotibial angle, femora antetorsion, and tibiofibular torsion: Cross-sectional study 
among healthy Japanese and Australian Caucasians. Clinical Anatomy. 2006; 19(1):59-67. doi 10.1002/ca.20170

[16] Rozzi SL, Lephart SM, Fu FH. Effects of muscular fatigue on knee joint laxity and neuromuscular characteristics of male and female athletes. Journal of Athletic Training. 1999; 34(2):106-114. PMCID: PMC1322898

[17] Riemann BL, Lephart SM. The sensorimotor system, part I: the physiologic basis of functional joint stability. Journal of Athletic Training. 2002; 37(1):71-90. doi: 10.1016/b978044306701-3.50052-9

[18] Gandevia SC. Neural control in human muscle fatigue: changes in muscle afferents, moto neurones and moto cortical drive. Acta Physiologica Scandinavica. 1998; 162(3):275-83 . doi: 10.1046/j.1365-201x.1998.0299f.x

[19] Rodenbusch TL, Ribeiro TS, Simão CR, Britto HM, Tudella E, Lindquist AR. Effects of treadmill inclination on the gait of children with Down syndrome. Research in Developmental Disabilities. 2013; 34(7):2185-190. doi: 10.1016/j. ridd.2013.02.014

[20] Cooke TD, Sled EA, Scudamore RA. Frontal plane knee alignment: a call for standardized measurement. Journal of Rheumatology. 2007; 34(9):1796-801. PMID: 17787049

[21] Madigan ML, Pidcoe PE. Changes in landing biomechanics during a fatiguing landing activity. Journal of Electromyography \& Kinesiology. 2003; 13(5):491-98. doi: 10.1016/s10506411(03)00037-3

[22] Ford KR, Myer GD, Hewett TE. Valgus knee motion during landing in high school female and male basketball players. Medicine \& Science in Sports \& Exercise. 2003; 35(10):1745-50. doi: 10.1249/01.mss.0000089346.85744.d9

[23] King D, Sigg J, Belyea B, Hummel CH, Buck M. Effects of fatigue on lower extremity joint kinematics during a stop and go landing task. Clinical Biomechistry. 2006; 36(5):393-410. 
